\title{
Lesión indurada de aparición en la infancia
}

\author{
María Flores Copete ${ }^{\mathrm{a}}$, José Manuel Azaña Defez y Ricardo Enrique Reolid Martíneza .
}

\footnotetext{
a Medicina de Familia y Comunitaria. Centro de Salud Zona 4. Albacete (España). ' Servicio de Dermatología y Venereología. Complejo Hospitalario Universitario de Albacete. Albacete (España).

\section{Correspondencia:}

María Flores Copete.

Centro de Salud Zona 4.

C/ Seminario, 4. C.P. 02006.

Albacete. España.

Correo electrónico:

florescopetemaria@gmail.com.

Recibido el 17 de noviembre de 2014.

Aceptado para su publicación el 18 de enero de 2015
}

\section{RESUMEN}

Presentamos un paciente de 13 años referido a la consulta de Dermatología por una lesión indurada, pigmentada, lineal en miembro inferior derecho. Tras su valoración clínica y con sospecha diagnóstica de morfea, se realizaron las exploraciones pertinentes para confirmar el diagnóstico e indicar el tratamiento específico.

Palabras Clave: Enfermedades del Tejido Conectivo. Esclerodermia Localizada. Morfea.

\section{ABSTRACT \\ Indurated lesion with onset in childhood}

We present a 13-year-old patient referred to the Dermatology consultation due to a lineal, indurated, pigmented lesion on the right lower limb. After clinical assessment and suspected diagnosis of morphea, relevant examinations were performed to confirm the diagnosis and to indicate the specific treatment.

Key words: Connective Tissue Diseases. Scleroderma, Localized. Morphea.

\section{INTRODUCCIÓN}

La morfea es una patología infrecuente, con una incidencia de 0,4-2,7 por 100.000 personas. Predomina en el sexo femenino y en la raza blanca, con una prevalencia similar en niños y adultos. Representa la forma más frecuente de esclerosis en la infancia, suponiendo aproximadamente el 0,2\% de las derivaciones a las consultas de dermatología pediátrica ${ }^{1}$.

La morfea o esclerodermia localizada es una enfermedad inflamatoria que conduce a la esclerosis de la piel y los tejidos subyacentes. Se diferencia de la esclerodermia sistémica por la ausencia de esclerodactilia, fenómeno de Raynaud, anomalías en los capilares del lecho ungueal y afectación de los órganos internos ${ }^{2}$. Su patogenia no está del todo aclarada, pero resulta de un disbalance entre la producción y la destrucción de colágeno ${ }^{3}$.

La morfea se puede clasificar según su presentación clínica en cinco variantes: en placa, generalizada, ampollosa, lineal y profunda ${ }^{1,2}$ 


\section{CASO CLÍNICO}

Niño de 13 años, con antecedentes personales de síndrome de Asperger, trastorno de déficit de atención e hiperactividad e hipotiroidismo, en tratamiento con metilfenidato, risperidona y levotiroxina. Fue enviado a la consulta de Dermatología por presentar una lesión en el miembro inferior derecho, de crecimiento progresivo, desde hacía

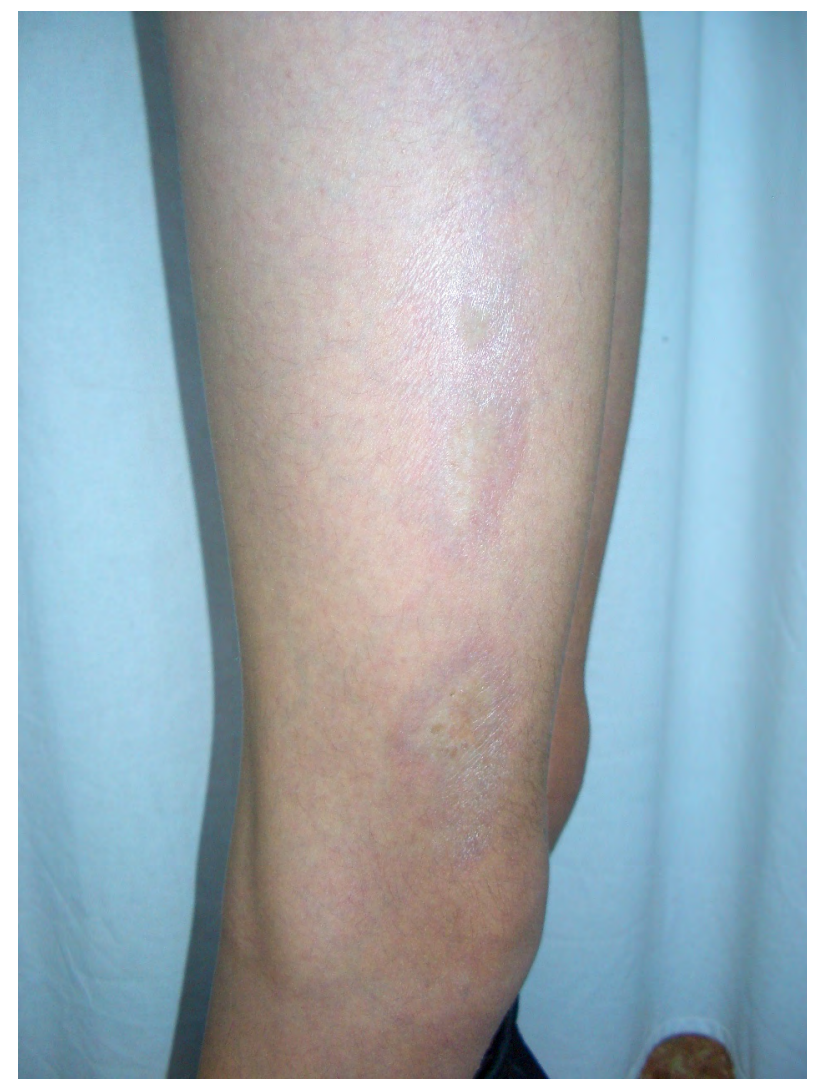

Figura 1. Placa esclerótica lineal extendiéndose a lo largo de la longitud del muslo

Se solicitó una analítica que incluía hemograma, bioquímica, anticuerpos antinucleares (ANA) y serologías, examen de orina, así como biopsia de la lesión. Los resultados fueron normales, con ANA, anti-Sm, anti-RNP, anti-SSA, anti-SSB, anti-Jo1 y anti-Scl70 negativos. La serología para Borrelia burgdorferi fue negativa y el análisis de orina no mostró anomalías. La histopatología fue compatible con el diagnóstico de morfea.

Ante una morfea lineal en la infancia sin afectación articular ni deformidad estética, se decidió iniciar tratamiento corticoideo tópico con propionato de un año. Era asintomática, sin presentar ninguna clínica como dolor o prurito. No había recibido ningún tratamiento, ni refería ningún factor desencadenante. En la exploración se observó una placa, indurada a la palpación, con un área discrómica central y un halo violáceo, de distribución lineal en el tercio medio-distal en la zona externa del muslo derecho (figuras 1 y 2). No presentaba otras lesiones cutáneas, ni clínica sistémica acompañante.

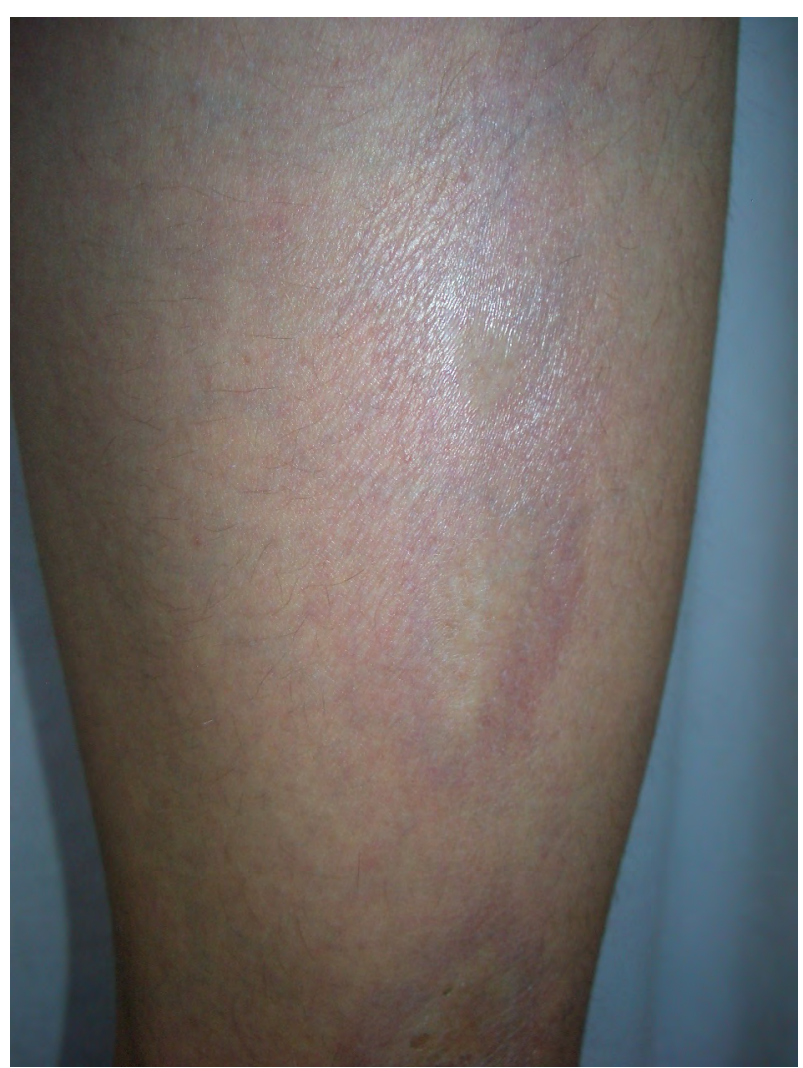

Figura 2. A mayor detalle se observa el centro esclerótico de coloración blanquecina y el anillo violáceo alrededor (lilac ring)

fluticasona, una aplicación al día durante 2 meses, seguido de calcipotriol tópico, también un aplicación diaria. Tras casi un año de seguimiento, las lesiones se han estabilizado.

\section{DISCUSIÓN}

De etiología desconocida, en la literatura se describen múltiples posibles desencadenantes asociados a su inicio, como traumatismos, vacunaciones, radioterapia, fármacos (bleomicina, D-penicilamina, 
bromocriptina, ibuprofeno o pentazocina) y agentes infecciosos como la Borrelia burgdorferi ${ }^{1,4,5}$.

La morfea lineal es una lesión única, unilateral y de distribución lineal, que con frecuencia asienta en las extremidades, la cara o el cuero cabelludo. Muchas veces estas lesiones lineales siguen las líneas de desarrollo embrionario (líneas de Blaschko), por lo que se ha propuesto que un posible mosaicismo genético sea el factor determinante de la distribución lineal. Pueden interferir en el crecimiento de la extremidad y ocasionar deformidades por la atrofia del músculo y el hueso subyacente, así como contracturas articulares. En superficie aparecen como bandas de piel deprimida, mal delimitadas, con trastornos de la pigmentación y en sus fases iniciales con un anillo violáceo (lilac ring) ${ }^{2}$.

En la morfea lineal, sobre todo cuando las lesiones asientan en el polo cefálico (tipo coup de sabre o hemiatrofia facial progresiva), se ha destacado la frecuencia con que asocian complicaciones neurológicas y oftalmológicas ${ }^{2}$, por lo que en estos casos se deberían realizar exploraciones complementarias para descartarlas ${ }^{6}$. Se debe solicitar hemograma, ANA, factor reumatoide, Scl-70, anticuerpos anticentrómero e inmunoglobulinas ${ }^{6}$, ya que podrían correlacionarse con la actividad de la enfermedad.

Dado que el diagnóstico de morfea es clínico, la biopsia se obtendría solo en los casos dudosos. En nuestro caso, dado el riesgo de evolución desfavorable (interferencia con el crecimiento) o necesidad de tratamiento sistémico (corticoides, metotrexato), se decidió confirmar el diagnóstico clínico con el histopatológico. La biopsia cutánea es un procedimiento invasivo pero de fácil ejecución; exploraciones complementarias como la ecografía cutánea o la termografía pueden resultar de utilidad, pero no las teníamos a nuestra disposición en ese momento.

Se debería establecer un diagnóstico diferencial con lesiones de distribución lineal en edad pediátrica, lesiones induradas y lesiones atróficas. Entre las primeras, destaca la hipermelanosis o la hipomelanosis nevoide lineal, trastornos de pigmentación congénitos o que se detectan en los primeros meses de vida, con un patrón lineal pero sin induración. También el vitíligo, en su forma lineal o segmentaria, y los trastornos de pigmentación postinflamatorios pueden seguir un trayecto lineal. Lesiones induradas como la fasceítis o fascitis eosinofílica, o el escleredema de Buschke, pero con características clínica distintas a la morfea lineal. En el liquen escleroso, considerado por algunos autores como una variante superficial de morfea, las lesiones cutáneas no presentan la morfología lineal, sino en forma de máculas ("gotas") o placas 5 . La atrofodermia idiopática de Pasini y Pierini consiste en un proceso cutáneo atrofiante sin fase inflamatoria y localización preferente en la espalda de mujeres jóvenes o de edad media.

La tendencia de la morfea a la resolución espontánea en muchos casos, así como su escasa incidencia y la no existencia de un marcador (clínico, analítico o prueba de imagen) de actividad, conllevan la ausencia de protocolos de tratamiento establecido ${ }^{1}$. La modalidad terapéutica elegida variará de acuerdo con la localización de las lesiones y la alteración funcional que estas produzcan 6 . El tratamiento tópico está indicado en aquellos pacientes con morfea en placas sin afectación articular y sin deformidad antiestética o funcional, como en nuestro caso. Entre las opciones disponibles encontramos los corticoides tópicos, el calcipotriol y el imiquimod. En los casos de enfermedad progresiva, morfea lineal a nivel facial o articular, falta de respuesta al tratamiento tópico o afectación subcutánea, muscular, ósea, articular, u otras manifestaciones extracutáneas, estaría indicado el tratamiento sistémico. El principal fármaco en este grupo sería el metotrexato, asociado en los primeros meses a corticoides sistémicos ${ }^{1}$.

Presentamos, en definitiva, un caso típico de morfea lineal en la infancia, de diagnóstico clínico sencillo, por lo que el médico de familia podrá realizar una primera aproximación diagnóstica al encontrarse ante una lesión de morfología lineal y con induración cutánea. Destacamos la necesidad de más estudios prospectivos que permitan definir cuál es el tratamiento más adecuado y los parámetros que permitan determinar de una manera objetiva la respuesta al mismo ${ }^{6}$.

\section{BIBLIOGRAFÍA}

1. Martínez Martínez ML, Azaña Defez JM, Rodríguez Váz- 
quez M, Faura Berruga C, López Villaescusa MT, Iñiguez de Onzoño L. Morfeas en la infancia: estudio retrospectivo. Med Cutan Iber Lat Am. 2012; 40 (1): 11-7.

2. Bielsa I. Actualización en la clasificación y el tratamiento de la esclerodermia localizada. Actas Dermosifiliogr. 2013; 104 (8): 654-666.

3. Fett N, Werth VP. Update on morphea: part I. Epidemiology, clinical presentation, and pathogenesis. J Am Acad Dermatol. 2011; 64 (2): 217-28.
4. Christen-Zaech S, Hakim MD, Afsar FS, Paller AS. Pediatric morphea (localized scleroderma): review of 136 patients. J Am Acad Dermatol 2008; 59 (3): 385-96.

5. Zulian F, Athreya BH, Laxer R, Nelson AM, Feitosa de Oliveira SK, Punaro MG, et al. Juvenile localized scleroderma: clinical and epidemiological features in 750 children. An international study. Rheumatology (Oxford). 2006; 45 (5): 614-20.

6. Laguna Argente C, García-Melgares Linares ML, Febrer Bosch I. Morfea infantil. Piel. 2009; 24 (1): 28-34. 